\title{
Anti-inflammatory and analgesic activity of ointment based on dense ginger extract (Zingiber officinale)
}

\author{
Iryna Kravchenko $^{1,2}{ }^{\circledR}$, Lidiya Eberle ${ }^{1}$, Mariia Nesterkina ${ }^{1,2^{*}}{ }^{\mathbb{D}}$, Alona Kobernik $^{1}$ \\ ${ }^{1}$ Department of Pharmaceutical Chemistry, I.I. Mechnikov Odessa National University, Odessa, 65082, Ukraine \\ ${ }^{2}$ Department of Organic and Pharmaceutical Technology of Odessa National Polytechnic University, 65044, Odessa, Ukraine
}

\section{A R T I C L E I N F O}

Article Type:

Original Article

Article History:

Received: 17 May 2018

Accepted: 24 January 2019

Keywords:

Zingiber officinale

Ginger extract

AITC-induced inflammation

Anti-inflammatory activity

Analgesic activity

Ointment

\begin{abstract}
A B S T R A C T
Introduction: Zingiber officinale (Zingiberaceae family) is traditionally used in alternative medicine to reduce pain from rheumatoid arthritis and osteoarthritis. Ginger is also often applied for stomach and chest pain, toothaches and as anti-inflammatory agent. The aim of this study is to investigate analgesic and anti-inflammatory activities of $Z$. officinale dense extract after its transdermal delivery using allyl isothiocyanate (AITC) induced model with further discussion of possible action mechanism of ginger phytoconstituents.

Methods: Inflammation was induced by subplantar injection to the plantar fasciitis (aponeurosis) of the hind limb of rats using $30 \mu \mathrm{L}$ AITC solution $(100 \mu \mathrm{g} / \mathrm{limb})$ in 1,2-propyleneglycol. The dynamics of changes of inflammatory process was evaluated before addition of the inflammation inducer and after 1,2, 3, 4, 6 and 24 hours of its injection for measuring the volume and the thickness of affected limb. Analgesic activity of ointments with ginger extract was examined using the model of AITC-induced pain.

Results: The most effective inhibition of the development of inflammation process was $0.025 \%$ ointment with ginger extract, and the highest anti-nociceptive effect was observed at the application of $0.05 \%$ ointment 10 minutes before pain inducer agent.

Conclusion: Zingiber officinale dense extract was revealed to possess significant antinociceptive and anti-inflammatory actions after its transdermal delivery. Since the pharmacological effects of ginger extract have been investigated on AITC-induced model, we may suggest the vital role of phytoconstituents binding to TRPA1 and TRPV1 ion channels as possible mechanism of action.
\end{abstract}

Implication for health policy/practice/research/medical education:

Zingiber officinale dense extract possesses significant anti-inflammatory and analgesic action and, consequently, might be used as an effective natural agent for pain and inflammation after transdermal delivery

Please cite this paper as: Kravchenko I, Eberle L, Nesterkina M, Kobernik A. Anti-inflammatory and analgesic activity of ointment based on dense ginger extract (Zingiber officinale). J Herbmed Pharmacol. 2019;8(2):126-132. doi: 10.15171/jhp.2019.20.

\section{Introduction}

The inflammatory process is the main pathogenetic element of many diseases and its specific localization and origin often defines the specificity of the disease $(1,2)$. Therefore, the regulation of the inflammatory process and development of new highly effective anti-inflammatory drugs to inhibit inflammation of the different genesis is one of the most relevant problems of modern medicine and pharmacology.

Nowadays, the complex therapy of the inflammatory process has a vast arsenal of the pharmacological drugs from the group of nonsteroidal anti-inflammatory drugs (NSAIDs) that can treat the inflammation process (3).
But despite the significant progress and achievements, the searching of the new and highly effective and less toxic anti-inflammatory drugs is the relevant task of the pharmacology. Compounds that are proposed as therapeutic medicine must have the broad spectrum of a therapeutic effect being harmless during the long-term use by having minimal side effects. Searching such antiinflammatory drugs among the plant raw materials is the most promising way in this regard.

Among such potential sources of medicinal products taken from traditional medicine, the ginger rhizome (Zingiber officinale Roscoe), which its history of use as a cure in different countries all over the globe has about 
2000 years, can also be attributed (4-6).

According to the literature, the ginger rhizome has a number of pharmacologically active substances such as magnesium, calcium and phosphorus salts, vitamins of group $B\left(B_{1}, B_{2}, B_{12}\right)$, ascorbic acid and vitamin $A$. Beside this, the rhizome contains iron, zinc, potassium, sodium, aluminium, chromium, germanium, asparagine, choline as well as amino acids essential for humans including tryptophan, threonine, leucine, methionine, valine, etc. Spicy tart flavour of $Z$. officinale is due to the presence of a large number of essential oils and phenol-like substances - gingerol, shogaol, capsaicin, etc (7-9).

The valuable pharmacological substances in Z. officinale rhizome give a reason to consider these raw materials as a promising cure to treat the inflammatory and painful conditions. This is due to their ability for specific blocking of the inflammation of the pain-sensitive receptors and ion channels TRP receptors in particular $(10,11)$.

Two representatives of the transient receptor potential channels family (TRP) - TRPA1 (ankyrin receptors) and TRPV1 (vanilloid receptors) play the main role in development of the nociceptive sensitization of nervous system, that might co-express in the peripheral sensory neurons $(12,13)$.

TRPV1 channels relate to polymodal receptors that are sensitive to high temperature (over $43^{\circ} \mathrm{C}$ ), changes in $\mathrm{pH}$ (acidosis and alkalosis), "endo vanilloids" (anandamide, metabolites of arachidonic acid, etc). On the other hand, TRPV1-channels are sensitive to action of such various "burning" plant compounds like capsaicin (from chilli pepper), piperine (the noxious compound of black pepper), gingerol and zingerone (from ginger), camphor and eugenol (from clove oil) (14).

Also, TRPA1-channels relate to polymodal nociceptors of various noxious stimuli including such "burning" chemicals as allyl isothiocyanate (AITC) that could be found in the mustard oil, thiosulfates that are in the garlic, cinnamic aldehyde and tear gas $(15,16)$. It is known that both of these channels are related to nonselective calciumpermeable cationic channels that take a part in the regulation of the intracellular concentration of calcium ions playing the signalling role in the nerve cells (17). It has been shown that TRPA1 and TRPV1 channels are capable of regulating each other and the activation one of them can enhance or inhibit the sensitivity of the another to the appropriate selective agonists $(14,18)$.

Since TRP receptors are the most important integrators of inflammatory and pain stimuli, they can be considered as a promising target during the treatment. Unlike traditional anti-inflammatory drugs that inhibit inflammatory processes or transmit pain signals, TRP antagonists prevent inflammation by blocking these receptors and integrators of pain stimuli on sensitive neurons (13). The aim of the study was to determine the anti-inflammatory and analgesic effect during the transdermal delivery of ointment with ginger extract as well as the establishment of a possible mechanism of antiinflammatory and analgesic activity.

\section{Materials and Methods}

AITC-induced acute inflammatory model

Investigation of anti-inflammatory activity of ointment with ginger extract was conducted on 50 white male nonlinear rats weighing 200-220 g using the model of AITC inflammation. The ointment based on dense ginger extract with $0.025 \%$ concentration that was recalculated as polyphenolic compounds was made for this study by using previous investigations that showed the best anti-inflammatory effect $(17,18)$. The ointment basis was consisted of polyethyleneglycol-1500, polyethyleneoxide-400 and 1,2-propyleneglycol in a ratio of 3:4:2, respectively. In comparison $5 \%$ ointment with ibuprofen ("Dolgit cream", Germany) has been used.

For this experiment 5 animal groups (10 rats each) have been made:

- Group 1: control (without treatment);

- Group 2: animals received preventive treatment of ointment with ginger extract two days before the start of the experiment and with subsequent treatment after phlogogen administration;

- Group 3: animals received applications of 5\% ibuprofen two days before the start of the experiment and with subsequent treatment after induction of inflammation;

- Group 4; animals were treated with ointment with ginger extract one day after the phlogogen administration;

- Group 5; animals were treated with reference medicine one day after the phlogogen administration.

Inflammation was induced by subplantar injection to the plantar fasciitis (aponeurosis) of the hind limb of rats using $30 \mu \mathrm{L}$ AITC solution $(100 \mu \mathrm{g} / \mathrm{limb})$ in 1,2-propyleneglycol. The dynamics of changes of inflammatory process was evaluated before addition of the inflammation inducer and after 1, 2, 3, 4, 6 and 24 hours of its injection by using electronic callipers YT-7201 ("YATO", Poland) for measuring the volume of affected limb, and limb thickness was measured using digital plethysmometer 37140 ("Ugo Basile", Italy).

Experiment was conformed to the rules of the "Guide for the Care and Use of Laboratory Animals" (NIH publication 86-23 revised 1985). Ointment with $Z$. officinale extract was applied to affected limb after each measuring and represented a percentage relative to output data. In addition, the indicator of anti-exudative activity was calculated, which, fundamentally, is a qualitative indicator of inhibition of development of inflammation in the experimental groups comparing to control group.

Anti-exudative activity (AA) was calculated by using following formula: 
$A A=\left(100-\left(\frac{P_{x i}-P_{\mathrm{int}}}{P_{\mathrm{int}}}\right)_{\substack{\text { research } \\ \text { groub }}}:\left(\frac{P_{x i}-P_{\mathrm{int}}}{P_{\mathrm{int}}}\right)_{\substack{\text { control } \\ \text { group }}}\right) \times 100$

Where: AA -anti-exudative activity;

$\mathrm{P}_{\mathrm{xi}}$ : value of limb volume after administration of inflammation inducer;

$\mathrm{P}_{\text {int }}$ : value of limb volume before administration of inflammation inducer.

\section{Antinociception testing}

Analgesic activity of ointments with ginger extract was examined using the model of AITC-induced pain. The experimental animals had subplantar injection of $20 \mu \mathrm{L}$ $0.5 \%$ AITC solution in 1,2-propyleneglycol.

The investigation of pain threshold and potential analgesic effect of ointments with ginger extract was conducted on white outbred mice weighing 18-22 g that were separated into 8 groups of 10 mice each. The first animal group was a control, and animals from group 2, 3, 4, 5, 6, 7 and 8 before AITC administration had application of ointment with concentration $0.0125 \%$ $0.025 \%-0.05 \%-0.1 \%-0.5 \%-1.0 \%-5.0 \%$ respectively. Each animal group was divided into 2 subgroups ( 5 mice each): the first subgroup had application of ointment 5 minutes before AITC administration, the second group 10 minutes before AITC administration.

After injection of AITC solution, each animal was placed in a transparent box immediately. The test animal was observed for 10 minutes after AITC administration and a total time spent by the animal on licking the affected limb was registered.

Analgesic activity of ointment was presented by the average latent time in the group and percentage of inhibiting of the pain response (IPR) that was calculated by using following formula:

$I P R=\frac{T_{\text {control }}-T_{\text {research }}}{T_{\text {control }}} \times 100$

where $\mathrm{T}$ - time of the pain response.
Statistical analysis of obtained results was made by using test (following Student's $t$-distribution) in order to establish the presence of statistically significant differences between the values of experimental and control animals.

\section{Results}

Anti-inflammatory activity of Zingiber officinale extract on AITC-induced model

Evaluation of obtained data during the determination of the volume of inflammatory foci by volumetric method indicated that volume of affected limbs both control and test groups at 1 hour after AITC injection increased by in average $45 \%$ by volume of the intact limb (Figure 1).

Transdermal application of ointment based on ginger extract and ibuprofen contributed to decreasing of inflammatory foci after the first therapeutic procedures. The tendency to decrease the volume of affected limbs in rats was observed since second hour of the experiment, in contract to control group, where the peak of inflammation was observed on third hour and exceeded intact values by 1.5 times.

Table 1 represented data of the measuring of the volume of affected limbs of rats from the test groups comparing to control group, that have not been treated, and the group that have been treated with reference medicine - ointment with ibuprofen.

During the experimental inflammation between values of groups that were treated with ointment with ginger extract and ibuprofen, the advantage of the reference medicine was observed until the third hour of the experiment, but the values did not differ from each other since the fourth hour.

On the sixth hour the anti-exudative activity was $69 \%$ for the experimental ointment, while anti-exudative activity for reference medicine was $62 \%$ in average.

After 24 hours of experiment, the volume of the animal limbs treated with developed ointment with ginger extract and ibuprofen reached intact values, while the morphological parameters of the control group of animals

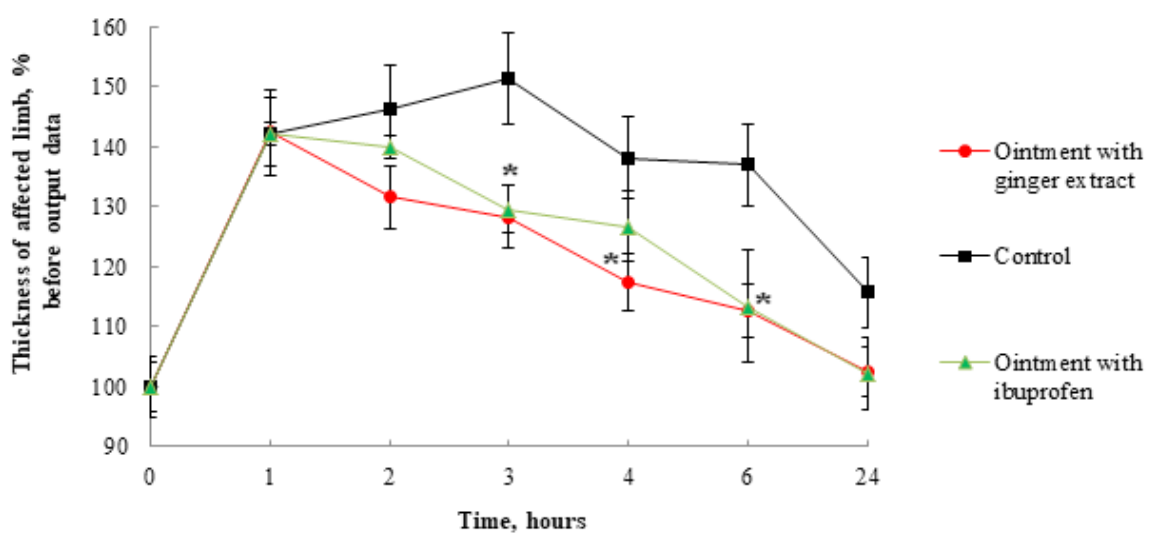

Figure 1. Anti-inflammatory activity of ointment based on ginger extract; treatment after phlogogen injection (width of edema of affected limbs of rats, $\%$ to intact; $M \pm m ; n=10) .{ }^{*} P<0.05$ comparing with control group. 
Table 1. Volume of affected limbs of rats $\left(\mathrm{cm}^{3}\right)$ and anti-exudative activity (\%) in terms of AITC-induced inflammation

\begin{tabular}{|c|c|c|c|c|c|c|c|c|}
\hline \multirow{3}{*}{ Experimental groups } & \multicolumn{5}{|c|}{ Average limb gain, $\mathrm{cm}^{3}$} & \multicolumn{3}{|c|}{ Anti-exudative activity, \% } \\
\hline & \multicolumn{8}{|c|}{ Observation time (hour) } \\
\hline & Before injection & 1 & 3 & 6 & 24 & 3 & 6 & 24 \\
\hline \multicolumn{9}{|c|}{ Preventive application of ointments ( 2 days before phlogogen injection) } \\
\hline Control & $0.68 \pm 0.05$ & $1.03 \pm 0.07$ & $1.11 \pm 0.09$ & $0.92 \pm 0.06$ & $0.84 \pm 0.05$ & - & - & - \\
\hline Ointment with ginger extract & $0.71 \pm 0.04$ & $0.88 \pm 0.07$ & $0.83 \pm 0.07 *$ & $0.75 \pm 0.05^{*}$ & $0.73 \pm 0.06$ & 73.23 & 84.04 & 88.03 \\
\hline Reference drug & $0.67 \pm 0.08$ & $0.98 \pm 0.09$ & $0.78 \pm 0.05^{*}$ & $0.75 \pm 0.06^{*}$ & $0.7 \pm 0.08$ & 74.04 & 66.17 & 80.97 \\
\hline \multicolumn{9}{|c|}{ Treatment in 1 hour after phlogogen injection } \\
\hline Ointment with ginger extract & $0.64 \pm 0.06$ & $0.96 \pm 0.08$ & $0.81 \pm 0.05^{*}$ & $0.71 \pm 0.04 *$ & $0.67 \pm 0.03^{*}$ & 60.47 & 69.01 & 80.08 \\
\hline Reference drug & $0.67 \pm 0.03$ & $1.02 \pm 0.06$ & $0.82 \pm 0.07^{*}$ & $0.76 \pm 0.09$ & $0.72 \pm 0.02$ & 64.60 & 61.94 & 68.28 \\
\hline
\end{tabular}

still exceeded intact values by $23.5 \%$.

Values of thickness changing of the affected limb were similar to dynamics volume values and within 1 hour after the inflammation induction, they tended to increase by an average of $43 \%$.

Applications of ointment with ginger extract and ibuprofen led to gradual inhibition of the inflammatory foci after the first use and after 2 hours of the experiment, the thickness of affected limb was 32\% and 39\% prospectively, output data, which exceeded the control group values, which continued to increase with a peak at $3^{\text {rd }}$ hour.

Subsequent applications of ointment based on ginger extract at third and sixth hour of experiment were the same as anti-inflammatory activity of ibuprofen and on average inhibited the inflammation development by $22 \%$ and $17 \%$ prospectively, comparing with control group. After 24 hours of the experiment results from the fourth and fifth groups reached intact values, while results from the control group exceeded intact values by $18 \%$.

Next stage of our study was the investigation of antiinflammatory activity of $0.025 \%$ ointment based on ginger extract with additional preventive application 2 days before phlogogen injection that possibly could determine the mechanism of interaction with ion-channel receptors.
In the animal groups, that had preventive application of ointment with ginger extract that was applied on the right hind limb, the strong anti-inflammatory activity was observed during the first hour after the injection of AITC agent. In this animal group the anti-exudative activity was manifested the inhibition of development of inflammatory foci comparing with other experimental groups.

At the first hour of the experiment in terms of additional preventive application of ointment with ginger extract the volume of affected limb increased by $24 \%$, and thickness by $28 \%$, while in the control group with preventive applications of reference medicine the volume increased by $49 \%$ in average, and thickness - by $43.5 \%$ comparing to output values (Figure 2).

It should be pointed that preventive application of ibuprofen 2 days before the start of the experiment did not influence on inhibition of inflammation, in contrast to the ointment with ginger extract, the anti-inflammatory effect of the reference medicine appeared immediately after inflammatory foci.

Subsequent treatment with $0.025 \%$ ointment, as it shown on Figure 2, led to a significant reduction of the volume and thickness of affected limb, in contrast to control group, where trend to inflammatory foci increasing was observed during second and third hours.

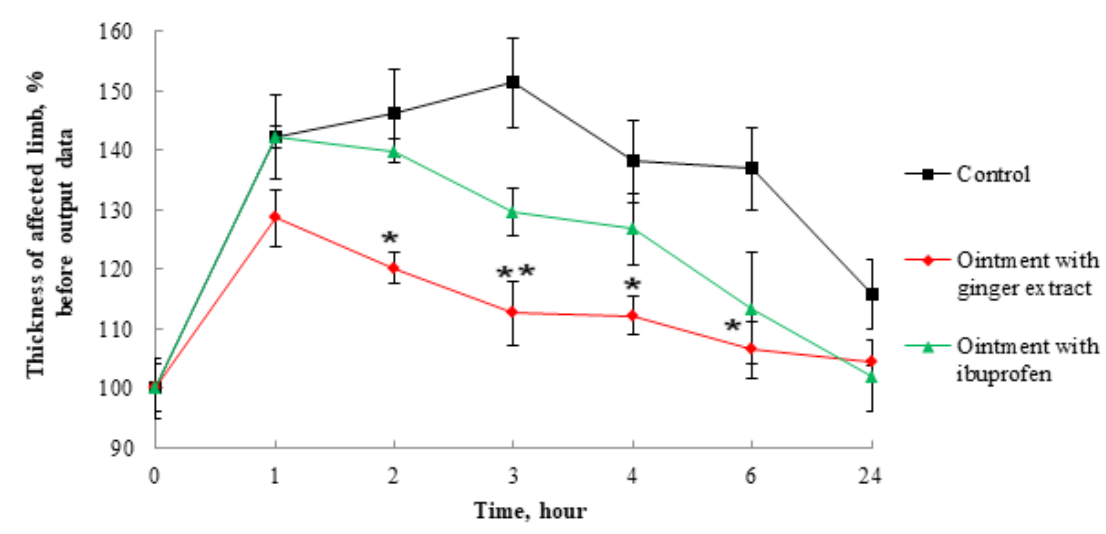

Figure 2. Anti-inflammatory activity of ointment based on ginger extract with additional preventive treatment (width of oedema of affected limbs of rats, \% to intact; $M \pm m ; n=10)$. Note. ${ }^{*} P<0.05 ;{ }^{* *} P<0.01$ comparing with control group. 
Analgesic effect of Zingiber officinale extract on AITCinduced model

The investigation of anti-nociceptive activity of ginger extract was studied by determining the pain threshold sensitivity on the model of AITC-induced pain in comparison with the reference medicine - benzocaine, the choice of which was due to its wide use in local anaesthesia as well as the ability of benzocaine to activate the TRP channels.

Investigation of analgesic activity of the ointment with ginger extract during the preventive application of the ointment for 5 and 10 minutes before the injection of the pain inductor showed that a more pronounced antinociceptive effect was observed in the application of ointments 10 minutes before the injection of AITC (Table 2).

It was found that use of $0.0125 \%$ and $0.025 \%$ of ointments based on a dense ginger rhizome extract 10 minutes before pain agent injection caused reduction of excitability of visceral nociceptors, which in turn stimulated a reduction in the licking time of hind limbs by animals by $30 \%$ and $36 \%$ respectively, whereas when applying $0.1 \%$ and $0.5 \%$ of the ointments, the inhibition of the pain reaction was $46.2 \%$ and $25.6 \%$, respectively.

The highest level of analgesic activity was observed during the application of $0.05 \%$ of the ointment, the duration of the pain response caused by the action of the chemical stimulus decreased to 70 seconds and 37 seconds and was not inferior to the reference medicine benzocaine.

It should be noted that the use of the tested ointments contributes to the proportional increase in IPR in the range of concentrations from $0.0125 \%$ to $0.05 \%$, and further increase of concentration of the extract causes the opposite effect. The highest level of IPR was found in the group, which were applied $0.05 \%$ ointment 10 minutes before AITC injection, it was 57\% compared with benzocaine - $45 \%$.

In terms of increase the concentration of the ointment to 1 and $5 \%$, the anaesthetic activity of the extract was absent, and the response time of the animals to the pain inducer was higher than the control group. The duration of the pain response was 85 seconds and 113 seconds (during the application 5 minutes prior), and 133 seconds and 153 seconds (during the application 10 minutes prior), respectively.

Summing all results of anti-inflammatory activity of the studied ointments with ginger extract on the model of AITC-induced inflammation, we can conclude that the most effective inhibition of the development of inflammation process was $0.025 \%$ ointment with ginger extract, and the highest anti-nociceptive effect is observed at the application of $0.05 \%$ ointment 10 minutes before pain inducer agent.

\section{Discussion}

The rhizome extract of $Z$. officinale was previously investigated as analgesic and anti-inflammatory agent after its intraperitoneal injection. Significant inhibition of carrageenan-induced inflammation as well as reduction in the number of acetic acid induced writhing was produced after extract administration (19). Ginger oil isolated by supercritical fluid extraction was also found to reduce painful sensations and inflammatory response in vivo (20). As known, $Z$. officinale contains a large number of phytochemicals such as shogaols, gingerols, diarylheptanoid, and proanthocyanidin (21). [6]-Gingerol, for instance, was examined on nociception induced by chemical stimuli and paw edema provoked by $\lambda$-carrageenin (22).

In the present work, dynamic of development of inflammatory and anti-nociceptive processes was investigated by using AITC-induced model for determining the complete picture of anti-inflammatory and analgesic activity of ginger extract after its transdermal delivery. Since it is known that AITC is an antagonist of TRPA1-channels, meaning one of the member in the pathway of pain signal transmission during inflammation and neuropathies, along with G-protein-coupled receptors, receptor tyrosine kinases (RTKs) and TRPV1,

Table 2. Analgesic activity of ointment with ginger extract on the model of AITC-induced test

\begin{tabular}{lcccc}
\hline \multirow{2}{*}{ Ointment concentration, \% } & \multicolumn{3}{c}{ The application time of the ointment before AITC injection } \\
\cline { 2 - 5 } & \multicolumn{2}{c}{$\mathbf{5}$ min prior } & \multicolumn{2}{c}{$\mathbf{1 0}$ min prior } \\
\cline { 2 - 5 } & Time response $(\mathbf{s})$ & IPR, \% & Time response (s) & IPR, \% \\
\hline Control & $86.5 \pm 2.5$ & - & $86.5 \pm 2.5$ & - \\
0.0125 & $81.6 \pm 1.8$ & 5.5 & $60.5 \pm 7.5^{*}$ & 30.0 \\
0.025 & $74.6 \pm 6.1$ & 13.7 & $55.3 \pm 1.7^{*}$ & 36.0 \\
0.05 & $69.6 \pm 2.7^{*}$ & 19.8 & $37.3 \pm 0.9^{*}$ & 56.8 \\
0.1 & $72.6 \pm 3.8$ & 15.9 & $46.5 \pm 0.5^{*}$ & 46.2 \\
0.5 & $76.6 \pm 1.4^{*}$ & 11.2 & $64.3 \pm 1.5^{*}$ & 25.6 \\
1.0 & $85.3 \pm 2.3$ & 1.6 & $132.7 \pm 6.7^{*}$ & - \\
5.0 & $112.6 \pm 7.6^{*}$ & - & $159 \pm 11.6^{*}$ & - \\
Benzocaine & $63.3 \pm 2.6^{*}$ & 26.9 & $44.3 \pm 1.2^{*}$ & 44.5 \\
\hline
\end{tabular}

$* P<0.05$ comparing to control. 
resulting the excitation of subpopulation of dissociated sensory neurons in the trigeminal ganglion that lead to development of inflammation and sense of pain.

From the literature, it is well known that oral use of ginger extract has a positive effect on dynamic of inflammation (6). The aim of this study was the determining of the level of inflammatory activity using ointment based on ginger extract, in terms of preventive transdermal application 2 days before phlogogen injection with subsequent treatment after phlogogen injection and treatment after inflammation induction in comparison with a control group and group with reference medicine. According to our data, it was found that $0.025 \%$ ointment based on ginger extract has a pronounced anti-inflammatory activity since it has been noticed that its previous preventive use contributed to blocking the development of inflammation during the first hour of the experiment and stimulated the reduction of the limb edema during subsequent applications with a return at sixth hour to initial values, in contrast to the control group and reference drug.

Since $Z$. officinale contains constituents differing in chemical structure, action mechanism of this extract involves various pharmacological targets. According to available data, the main ginger components - 10-gingerol, 8-shogaol and 10-shogaol - inhibit strongly COX-2 (8) or block the activity of both COX-1 and COX-2 (23).

On the other hand, ginger extract and its pungent constituents were found to modulate TRPC5 and TRPA1 currents and act as activators of TRPV1 channels (24). In the present research, inflammatory and painful processes were induced by powerful irritant and TRP channel activator - AITC. Despite the fact that AITC is generally accepted to modify TRPA1 ion channels via covalent modification of cysteine residues, AITC was also found to involve in binding process to vanilloid receptor 1 (TRPV1) (25). Taking into account that TRP channels are expressed both in nerve endings of skin and non-neuron cells (epidermal keratinocytes and melanocytes) (26), topical application of $Z$. officinale extract is expedient. Thus, the analgesic and anti-inflammatory effect of ginger may be partly due to interaction between its phytoconstituents and TRP subfamily members A1 and V1.

\section{Conclusion}

In conclusion, $Z$. officinale dense extract was revealed to possess significant antinociceptive and antiinflammatory action after its transdermal delivery. Since the pharmacological effect of ginger extract has been investigated on AITC-induced model, we may suggest the vital role of phytoconstituents binding to TRPA1 and TRPV1 ion channels as possible mechanism of action.

\section{Author Contributions}

IK conceived and designed the experiment; LE and AK performed the experiments; IK, LE and AK analyzed the data; IK, LE, AK and MN wrote the paper. All authors read and confirmed publication of the paper.

\section{Conflicts of Interest}

The authors declare no conflict of interest.

\section{Ethical considerations}

Ethical issues including plagiarism, misconduct, data fabrication, falsification, double publication or submission have been carefully checked by authors.

All experimental procedures conform to the guiding principles for research as recommended by "Guide for the Care and Use of Laboratory Animals" (NIH publication 86-23 revised 1985).

\section{Funding/Support}

None.

\section{References}

1. Okin D, Medzhitov R. Evolution of inflammatory diseases. Curr Biol. 2012;22:733-740. doi: 10.1016/j.cub.2012.07.029.

2. Chen L, Deng $\mathrm{H}$, Cui $\mathrm{H}$, et al. Inflammatory responses and inflammation-associated diseases in organs. Oncotarget. 2018;9:7204-18. doi: 10.18632/oncotarget.23208.

3. Boiko YA, Kravchenko IA, Shandra AA. Extraction, identification and anti-inflammatory activity of carotenoids out of Capsicum anuum. J Herbmed Pharmacol. 2017;6:10-15.

4. Kumar S, Sabiha M, Nisha R, Ankita K, Anil D. Evaluation of Zingiber officinale and Curcuma longa rhizome as a crude drug from their ethanolic extract. Int Res J Pharm. 2013;4:74-6. doi: 10.7897/2230-8407.041217.

5. Hasan HA, Raauf AM, Razik BM. Chemical composition and antimicrobial activity of the crude extracts isolated from Zingiber Officinale by different solvents. Pharmaceut Anal Acta. 2012;3:34-37. doi: 10.4172/2153-2435.1000184.

6. Mashhadi NS, Ghiasvand R, Askari G, Hariri M, Darvishi L, Mofid MR. Anti-Oxidative and anti-Inflammatory effects of ginger in health and physical activity: review of current evidence. Int Prev Med. 2013;4:12-17.

7. Dugasani SB, Pichika MR, Nadarajah VD. Comparative antioxidant and anti-inflammatory effects of [6]-gingerol, [8]-gingerol, [10]-gingerol and [6]-shogaol. J Ethnopharmacol. 2009;127:515-20. doi: 10.1016/j. jep.2009.10.004.

8. van Breemen RB, Tao Y, Wenkui L. Cyclooxygenase-2 inhibitors in ginger (Zingiber officinale). Fitoterapia. 2011; 82: 38-43. doi: 10.1016/j.fitote.2010.09.004.

9. Hazan A, Kumar R, Matzner H, Priel A. The pain receptor TRPV1 displays agonist-dependent activation stoichiometry. Sci Rep. 2015; 5: 1-13. 10.1038/srep12278.

10. Brito R, Sheth S, Mukherjea D. TRPV1: a potential drug target for treating various diseases. Cells. 2014;3:517-45. doi: $10.3390 /$ cells3020517.

11. Veronesi B, Oortgiesen M. The TRPV1 receptor: target of toxicants and therapeutics. Toxicol Sci. 2006;89:1-3.

12. Jara-Oseguera A, Simon SA, Rosenbaum T. TRPV1: on the road to pain relief. Curr Mol Pharmacol. 2008;1:255-269. 
13. Meotti FC, Lemos de Andrade E, Calixto JB. TRP modulation by natural compounds. Handbook Exp Pharmacol. 2014;223:1177-238. doi: 10.1007/978-3-31905161-1_19.

14. Cevikbas F, Wang X, Akiyama T, Kempkes C4, Savinko T, Antal A, et al. A sensory neuron-expressed IL-31 receptor mediates $\mathrm{T}$ helper cell-dependent itch: Involvement of TRPV1 and TRPA1. J Allergy Clin Immunol. 2014;133:44860. doi:10.1016/j.jaci.2013.10.048.

15. Liu B, Escalera J, Balakrishna S, Fan L, Caceres AI, Robinson E, et al. TRPA1 controls inflammation and pruritogen responses in allergic contact dermatitis. FASEB J. 2013; 27: 3549-3563. 10.1096/fj.13-229948.

16. Eberhardt MJ1, Filipovic MR, Leffler A, de la Roche J, Kistner K, Fischer MJ, et al. Methylglyoxal activates nociceptors through transient receptor potential channel A1 (TRPA1): a possible mechanism of metabolic neuropathies. J Biol Chem. 2012;287:28291-306. doi: 10.1074/jbc. M111.328674.

17. Kravchenko I, Kobernik A, Eberle L. Optimization of extraction methods for total polyphenolic compounds obtained from rhizomes of Zingiber officinale. Trends in Phytochem Res. 2018;2:37-42.

18. Kravchenko I, Kobernik A, Eberle L. Anti-inflammatory activity of solid ginger extract (Zingiber officinale) under transdermal introduction. J Pharmacol Drug Toxicol. 2017;6:43-9.
19. Raji I, Udon US, Oluwadara OO. Anti-inflammatory and analgesic properties of the rhizome extract of Zingiber Officinale. Afr J Biomed Res. 2002;5:121-4.

20. Jia Y, Zhao J, Zhang L, et al. Analgesic and anti-inflammatory effects of ginger oil. Chinese Herbal Medicines. 2011;3:1505.

21. Al-Nahain A, Jahan R, Rahmatullah M. Zingiber officinale: a potential plant against rheumatoid arthritis. Arthritis. 2014;2014:159089. doi: 10.1155/2014/159089.

22. Young H-Y, Luo Y-L, Cheng H-Y, Hsieh W-C, Liao J-C, Peng W-H. Analgesic and anti-inflammatory activities of [6]-gingerol. J Ethnopharmacol. 2005; 96: 207-210. doi: 10.1016/j.jep.2004.09.009.

23. Grzanna R, Lindmark L, Frondoza CG. Ginger - an herbal medicinal product with broad anti-inflammatory actions. J Med Food. 2005;8:125132. doi: 10.1089/jmf.2005.8.125.

24. Vriens J, Nilius B, Vennekens R. Herbal compounds and toxins modulating TRP channels. Curr Neuropharmacol. 2008;6:79-96. doi: 10.2174/157015908783769644.

25. Gees M, Alpizar YA, Boonen B, Sanchez A, Everaerts W, Segal A, et al. Mechanisms of transient receptor potential vanilloid 1 activation and sensitization by allyl isothiocyanate. Mol Pharmacol. 2013;84:325-334. doi: 10.1124/mol.113.085548.

26. Ho JC, Lee CH. TRP channels in skin: from physiological implications to clinical significances. Biophysics. 2015;11:17-24. doi: 10.2142/biophysics.11.17. 